\title{
Affordance-based Design of Physical Interfaces for Ubiquitous Computing Environments
}

\author{
Jennifer Sheridan, Gerd Kortuem \\ Computing Department \\ Lancaster University \\ \{sheridaj, kortuem\} @ comp. lancs.ac.uk
}

\begin{abstract}
Physical interfaces have been proposed as a way to realize natural interactions with ubiquitous computing environments. The successful design of such interfaces requires design approaches that integrate aspects of our world which are usually treated separately in traditional system development approaches. This paper describes a design approach based on Gibson concept of affordance.
\end{abstract}

\section{Introduction}

Ubiquitous computing promises a computing infrastructure that seamlessly aids users in accomplishing their tasks and that renders the actual computing devices and technology virtually invisible and distraction-free. Mark Weiser formulated this vision by describing a computer "so imbedded, so fitting, so natural, that we use it without even thinking about it" [1]. One way of realizing this vision is by building task-specific information appliances [2] and physical computer interfaces [3,4,5]. In fact, in recent years a number of toolkits kits for physical computer interfaces have appeared, including Phidgets [6], iSuff [7], SWEETPEA [8], Papier-Mâché [9] and MetaCricket [10]. While these toolkits provide adequate guidance during the construction phase of a physical interface, they do not provide any help in how to design a usable and intuitive physical interface.

The key idea of physical interfaces is to capitalize on our familiarity with the physical world. The design of such interfaces requires design approaches that incorporate different aspects of our world which are usually treated separately in traditional system development approaches. In particular, the actual physical form of an interface has rarely been considered in the context of physical interfaces and ubiquitous computing. This is in stark contrast to the fields of ergonomics and industrial design which have long recognized the importance of physical form for creating usable and appealing artefacts and products. One of the first researchers to systematically investigate the relationship between physical objects and people was the perceptual psychologist $\mathrm{J}$. J. Gibson who introduced the theory of affordance [11]. This theory states that physical objects suggest by their shape and other attributes what actors can do with them. Yet, theories of affordance are predominantly used as analytical tool and applied after the design stage. We believe that a thorough investigation of object affordance should be a key component of the physical interface design process.

\section{Affordance-based Design Method}

We are working on an affordance-based design method for physical interfaces that focuses on three key dimensions, namely affordance, capability and control.

- Affordance: We propose that the design of physical interfaces should begin with an investigation of the object itself in relation to human perception and motor skills, because physical form fundamentally shapes the kinds of interactions users can perform. The goal is to identify the types of actions humans can perform on an object. We formulate the results of this study as non-verbal dynamics, a vocabulary of significant object-specific manipulations such as gripping, squeezing, rubbing and rotating.

- Capability: The second component in the design process is the investigation and specification the technical capabilities of the interface artefact in terms of sensing and actuation. Technological capabilities affects which object manipulations can be recognized and how feedback is realized. As well, technology affects the form or physical properties of the device, such as shape and size.

- Control: The ultimate purpose of a physical interface is determined by the control it gives users over an application or service. For example, a mobile phone interface needs to provide controls for initiating and terminating a call. The set of controls needs to be mapped to non-verbal dynamics and realized by object capabilities. 


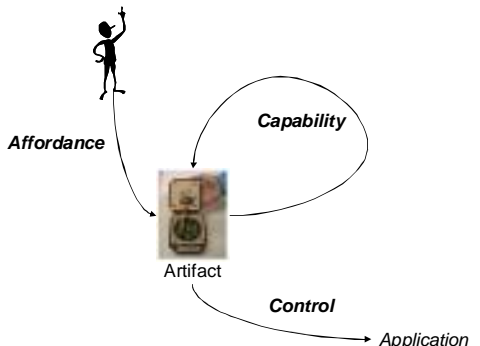

Figure 1. Design Method Overview
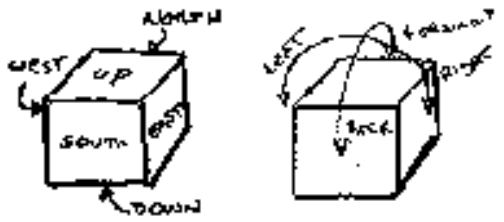

Figure 2. Cube Manipulations

We believe that these design dimensions are not separable, but must be investigated together. Understanding and applying the relationships between these three dimensions is the key to modelling and creating computer interfaces that are useful and appropriate for the ubiquitous world.

\section{Case Study}

We have applied our design method to the design of a physical interface for a mobile phone. As physical form we chose a six-sided cube. The goal was to design an interface that makes use of basic object manipulation skills rather than buttons. The cube interface is only used for input; output is presented on a separate (wristmounted or heads-up) display. The decision to use a cube-shaped object was based on the fact there is extensive prior work on using cubes as tangible or physical interface objects (for example BUILD-IT [12], Toolstone [13], Flip Bricks [14], ActiveCube [15], Cognitive Cubes [16], Navigational Blocks [17] and CUBIK [21]). While many experiments use six-sided objects, very few explain why they chose this particular physical form over others, and none reports on experimentation with other shapes. One of the reasons cubes are attractive is that most people have an intuitive and immediate understanding of how it can be manipulated [17]. While some studies report that cubes provide a higher flexibility in operations [19, 20] there is little discussion about why cubes are particularly well suited for this capability.

Early results suggest that following our approach leads to usable and desirable physical interfaces. Furthermore, it assists designers in evaluating and identifying design problems and suggests entry points for systematic redesign. Although we have applied our approach only to cubes and cube-like shapes, we believe that it applies to other shapes as well.

\section{References}

1. Mark Weiser, “Creating the Invisible Interface,” Proc. 7th Ann. ACM Symp. User Interface Software and Technology, ACM Press, 1994. 2. Don Norman. The Invisible Computer.

3. H. Ishii and B. Ullmer, "Tangible Bits: Towards Seamless Interfaces between People, Bits and Atoms," Proc. of CHI '97, ACM Press, 1997

4. P. Wellner, "Interacting with paper on the DigitalDesk," Communications of the ACM, 1993, vol. 36, no. 7, pp. 87-96.

5. Ullmer, B., Ishii, H. and Glas, D.(1999) mediaBlocks: Physical Containers, Transports, and Controls for Online Media. Proc. of the 25th Annual Conference on Computer Grapics.

6. Greenberg, S. and Fitchet, C. (2001) Phidgets: Easy Development of Physical Interfaces through Physical Widgets. Proc UIST 2001.

7. Anderson Ballagas, R., Ringel, M, Stone, M., Borchers, J, "iStuff: A Physical User Interface Toolkit for Ubiquitous Computing Environments", CHI 2003.

8. Kaminsky, M., Dourish, P., Edwards, K. LaMarca, A., Salisbury, M. and Smith, I. (1999) SWEETPEA: Software Tools for Programmable Embodied Agents. Proceedings of ACM CHI 99 Conference on Human Factors in Computing Systems, 144-151.

9. Scott R. Klemmer, Jack Li, James Lin, and James A. Landay, Papier-Mâché: Toolkit Support for Tangible Input. CHI Letters, Human Factors in Computing Systems: CHI2004. 6(1).

10. F. Martin, B. Mikhak, and B. Silverman. MetaCricket: A designer's kit for making computational devices. IBM Systems Journal, Volume 39, Numbers $3 \& 4,2000$.

11. J. J. Gibson, "The Theory of Affordances," The Ecological Approach to Visual Perception, Lawrence Erlbaum, Hillsdale, 1979.

12. M. Fjeld, M. Bichel and M. Rauterberg, "BUILD-IT: An Intuitive Design Tool Based on Direct Object Manipulation," In Gesture and Sign Language in Human-Computer Interaction, vol. 1371, Wachsmut and Frohlich, eds. Springer-Verlag, 1998, pp. $297-308$.

13. J. Rekimoto, B. Ullmer, and H. Oba, "ToolStone: Effective use of the physical manipulation vocabularies of input devices," Proc. of UIST'02, 2000, pp. 109-117.

14. G. Fitzmaurice, H. Ishii and W. Buxton, "Bricks: Laying the Foundations for Graspable User Interfaces," Proc. of CHI'95, 1995.

15. Y. Kitamura, Y. Itoh, T. Masaki and F. Kishino, "ActiveCube: A Bi-directional User Interface using Cubes," Fourth International Conference on Knowledge-Based Intelligent Engineering Systems \& Allied Technologies, 2000, pp. 99-102.

16. E. Sharlin, Y. Itoh, B. Watson, Y. Kitamura, L. Liu and S. Sutphen, "Cognitive Cubes: A Tangible User Interface for Cognitive Assessment," Proc. of CHI'02, 2002, pp. 347-354.

17. K. Camarata, E. Yi-Luen Do, B. R. Johnson and M. D. Gross, "Navigational Blocks: Navigating information space with tangible media," Proc. of IUI'02, 2002, pp. 31-38.

19. B. Ullmer, H. Ishii and D. Glas, mediaBlocks: Physical Containers, Transports, and Controls for Online Media, Proc. of SIGGRAPH'98.

20. J. Rekimoto, “Tilting operations for small screen interfaces,” Proc. of UIST'96, 1996, pp. 167-168.

21. S. Lertsithichai and M. Seegmiller, “CUBIK: A Bi-Directional Tangible Modeling Interface,” Proc. of CHI’02, pp. $393-402$. 